Proc. XII Int. School on Theoretical Physics — Symmetry and Structural Properties of Condensed Matter

\title{
Andreev Conductance through a Quantum Dot Strongly Coupled to Ferromagnetic and Superconducting Leads
}

\author{
K.P. WÓJCIK* AND I. WEYMANN \\ Faculty of Physics, Adam Mickiewicz University, Umultowska 85, 61-614 Poznań, Poland
}

\begin{abstract}
We analyze the Andreev conductance through a quantum dot strongly coupled to one ferromagnetic and one superconducting lead. The transmission due to the Andreev reflection is obtained from the numerical renormalization group method. We show that at low temperatures, depending on the dot level position, the Andreev conductance exhibits a peak at zero bias due to the Kondo effect, which can be split by the exchange field due to spin-dependent coupling to ferromagnetic lead.
\end{abstract}

DOI: 10.12693/APhysPolA.132.143

PACS/topics: 73.23.-b, 72.15.Qm, 74.45.+c, 72.25.-b

\section{Introduction}

At the interface between the superconductor $(\mathrm{S})$ and the normal metal $(\mathrm{N})$, the conventional low-bias current is suppressed, due to the energy gap in the superconducting single-electron spectrum. However, a process called the Andreev reflection is possible [1], in which an electron tunneling from $\mathrm{N}$ into $\mathrm{S}$ couples with another electron from $\mathrm{N}$ to form a Cooper pair in $\mathrm{S}$, thus leaving a hole in $\mathrm{N}$, which is reflected back. In a confined geometry, e.g. when $\mathrm{S}$ is coupled to $\mathrm{N}$ through a quantum $\operatorname{dot}(\mathrm{QD})$, such Andreev reflection processes are also possible, leading to the formation of bound states at the S-QD boundary [2], and giving rise to a finite current flowing through the system $[3,4]$. In the strong coupling regime, the Coulomb correlations on QD may give rise to another interesting phenomenon, namely the Kondo effect $[5,6]$. In the proximity with $\mathrm{S}$, the Kondo peak can be enhanced and the conductance can reach the value $4 e^{2} / h[7,8]$. However, this occurs only for the Coulomb correlations properly adjusted to the asymmetry in the couplings to $\mathrm{N}$ and $\mathrm{S}$ lead [9].

In this paper we address a specific question of how the Andreev current is modified in a hybrid, stronglycorrelated quantum dot device with one ferromagnetic (F) and one superconducting (S) electrode. When QD is coupled to ferromagnetic leads, the renormalization of the dot's level due to the coupling becomes spindependent, giving rise to an effective exchange field, defined as the difference between spin-up and spin-down dot's level. The exchange field splits and suppresses the Kondo resonance [10-12]. The exchange-field-related effects have recently been observed in hybrid quantum dots with $\mathrm{F}$ and $\mathrm{S}$ leads [13]. Here, we thus analyze the transport properties of such hybrid systems, paying special attention to the interplay of three relevant phenomena:

*corresponding author; e-mail: kpwojcik@amu.edu.pl the superconducting proximity effect, the Kondo correlations and the exchange field. In the following we put the Boltzmann constant $k_{B} \equiv 1$.

\section{Model and method}

The illustration of the considered system is presented in Fig. 1. The ferromagnetic lead is modeled via freeelectron Hamiltonian. Denoting by $c_{\mathrm{F} \boldsymbol{k} \sigma}$ the annihilation operator of an electron with momentum $\boldsymbol{k}$ and spin $\sigma$ of energy $\varepsilon_{\mathrm{F} \boldsymbol{k} \sigma}$, it is given by $H_{\mathrm{F}}=\sum_{\boldsymbol{k} \sigma} \varepsilon_{\mathrm{F} \boldsymbol{k} \sigma} c_{\mathrm{F} \boldsymbol{k} \sigma}^{\dagger} c_{\mathrm{F} \boldsymbol{k} \sigma}$.

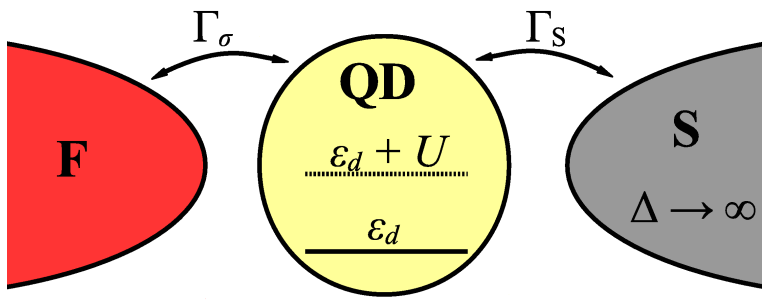

Fig. 1. Schematic of the system: quantum dot, with level energy $\varepsilon_{d}$ and Coulomb correlations $U$, is coupled to one ferromagnetic and one superconducting lead.

We consider a single-level QD with level energy $\varepsilon_{d}$ and Coulomb correlations $U$. Its Hamiltonian is given by $H_{\mathrm{QD}}=\varepsilon_{d} d_{\sigma}^{\dagger} d_{\sigma}+U d_{\uparrow}^{\dagger} d_{\uparrow} d_{\downarrow}^{\dagger} d_{\downarrow}$, where $d_{\sigma}$ annihilates $\sigma$-spin electron in the dot.

The superconductor is described with the mean-field form of the BCS Hamiltonian [14]:

$$
H_{\mathrm{S}}=\sum_{\boldsymbol{k} \sigma} \varepsilon_{\mathrm{S} \boldsymbol{k}} c_{\mathrm{S} \boldsymbol{k} \sigma}^{\dagger} c_{\mathrm{S} \boldsymbol{k} \sigma}-\Delta\left(c_{\mathrm{S} \boldsymbol{k} \uparrow}^{\dagger} c_{\mathrm{S}-\boldsymbol{k} \downarrow}^{\dagger}+c_{\mathrm{S}-\boldsymbol{k} \downarrow} c_{\mathrm{S} \boldsymbol{k} \uparrow}\right) .
$$

Here, $c_{\boldsymbol{s} \boldsymbol{k} \sigma}$ is the annihilation operator of a spin- $\sigma$ electron with momentum $\boldsymbol{k}$ and energy $\varepsilon_{\mathrm{S} \boldsymbol{k}}$. The superconducting order parameter $\Delta$ is assumed to be real, which is justified by the fact that we consider only one superconductor and thus phase is irrelevant.

The coupling between the dot and the leads is described by the tunneling Hamiltonian $H_{\mathrm{T} r}=$ $\sum_{\boldsymbol{k} \sigma} V_{r \boldsymbol{k} \sigma}\left(c_{r \boldsymbol{k} \sigma}^{\dagger} d_{\sigma}+d_{\sigma}^{\dagger} c_{r \boldsymbol{k} \sigma}\right)$, with $r=\mathrm{F}$ for the ferromagnet and $r=\mathrm{S}$ for the superconductor. In the wide 
band limit, the $\boldsymbol{k}$-dependence of hoping matrix elements $V_{r \boldsymbol{k} \sigma}$ and the energy dependence of the densities of states in the leads can be neglected. Thus, the strength of the coupling (dot level half-width) between the dot and the leads can be written as $\Gamma_{\sigma}=\pi \rho_{\mathrm{F} \sigma}\left|V_{\mathrm{F} \sigma}\right|^{2}=(1+\sigma p) \Gamma$, for the coupling to ferromagnet, and $\Gamma_{\mathrm{S}}=\pi \rho_{\mathrm{S}}\left|V_{\mathrm{S}}\right|^{2}$, for the coupling to superconductor. Here, $\rho_{\mathrm{S}}$ is the density of states of superconductor in the normal state, $\rho_{F \sigma}$ is the spin-dependent density of states of ferromagnet and $p$ denotes its spin polarization.

The total Hamiltonian is the sum of the aforementioned parts

$$
H=H_{\mathrm{F}}+H_{\mathrm{TF}}+H_{\mathrm{QD}}+H_{\mathrm{TS}}+H_{\mathrm{S}} .
$$

Since we focus only on the Andreev current, we take the limit of infinite superconducting energy gap, $\Delta \rightarrow \infty$. Then, the dot coupled to superconductor can be described by an effective Hamiltonian [9, 15]:

$$
\begin{aligned}
& H_{\mathrm{QD}}+H_{\mathrm{S}}+H_{\mathrm{TS}} \approx H_{\mathrm{QD}}^{*} \equiv H_{\mathrm{QD}} \\
& +\Gamma_{S}\left(d_{\uparrow}^{\dagger} d_{\downarrow}^{\dagger}+d_{\downarrow} d_{\uparrow}\right),
\end{aligned}
$$

where the internal degrees of freedom of $\mathrm{S}$ have been integrated out. The eigenstates of $H_{\mathrm{QD}}^{*}$ are two doublet states with singly-occupied QD of energy $\varepsilon_{d}$ and two singlet states, being the combination of empty and doubly occupied QD states, of energies

$$
E_{A \pm}=\varepsilon_{d}+U / 2 \pm \sqrt{\left(\varepsilon_{d}+U / 2\right)^{2}+\Gamma_{S}^{2}} .
$$

The excitation energies between the doublet and singlet states define the Andreev bound state energies, through which transport takes place.

The current flowing through the system due to the Andreev reflection processes can be expressed as

$$
I_{\mathrm{A}}=\frac{e}{h} \int \mathcal{T}_{\mathrm{A}}(\omega)[f(\omega-e V)-f(\omega+e V)] \mathrm{d} \omega,
$$

where the Andreev transmission is given by [16]:

$$
\mathcal{T}_{\mathrm{A}}(\omega)=4\left(1-p^{2}\right) \Gamma^{2} \sum_{\sigma}\left|\left\langle\left\langle d_{\sigma} \mid d_{\bar{\sigma}}\right\rangle\right\rangle_{\omega}\right|^{2},
$$

with $f(\omega)$ being the Fermi-Dirac distribution function, $V$ - the bias voltage, $\bar{\sigma} \equiv-\sigma$, and $\left\langle\left\langle d_{\sigma} \mid d_{\bar{\sigma}}\right\rangle\right\rangle_{\omega}$ denoting the Fourier transform of corresponding retarded Green's function. The last quantity is calculated with the aid of the numerical renormalization group (NRG) procedure $[17,18]$. Having obtained the imaginary part of $\left\langle\left\langle d_{\sigma} \mid d_{\bar{\sigma}}\right\rangle\right\rangle_{\omega}$ as a set of discrete delta peaks from the NRG data, we use the method proposed in Ref. [19] to broaden them appropriately into a continuous function. Then, we calculate the real part through the KramersKronig relations and in this way obtain $\left|\left\langle\left\langle d_{\sigma} \mid d_{\bar{\sigma}}\right\rangle\right\rangle_{\omega}\right|^{2}$. We assume that $\left|\left\langle\left\langle d_{\sigma} \mid d_{\bar{\sigma}}\right\rangle\right\rangle_{\omega}\right|^{2}$ is independent of $T$ and the temperature dependence of the current and differential conductance can be obtained through the Fermi functions in Eq. (5). Actually, to be as concise as possible, we consider only three temperatures: $T=0.001 U$, which is below the Kondo temperature $T_{K} \approx 0.008 U$ (defined for $\varepsilon_{d}=-0.5 U$ and $\left.p=0\right), T=0.1 U$, at which the Kondo physics is suppressed, and the intermediate case, $T=0.01 U$.

\section{Results and discussion}

As mentioned before, when quantum dot is coupled to ferromagnetic lead, the effective exchange field can split the dot's level. In the case of hybrid dots with $\mathrm{F}$ and $\mathrm{S}$ leads, such exchange field was recently estimated perturbatively in the limit of $\Delta \rightarrow \infty$ [20], and was found to be a function of the Andreev bound state energies. It was shown that the magnitude and sign of the level splitting due to the exchange field can be tuned by changing the dot level position, with vanishing exchange field at the particle-hole symmetry point, $\varepsilon_{d}=-U / 2$. For this reason, to determine the effects solely due to the exchange field, in the following we study the level dependence of the differential conductance $\mathrm{d} I_{\mathrm{A}} / \mathrm{d} V$ due to the Andreev current for two dot level configurations: $\varepsilon_{d}=-0.5 \mathrm{U}$ and $\varepsilon_{d}=-0.33 U$. The consequences of the presence of the exchange field are absent in the former configuration. Moreover, we compare and contrast $\mathrm{d} I_{\mathrm{A}} / \mathrm{d} V$ obtained in the case of normal metallic lead (Fig. 2) with that obtained in the case of ferromagnetic lead with finite polarization $p=0.5$ (Fig. 3). We use the half-width of the band as the energy unit, $D \equiv 1$, and assume the following parameters: $U=0.1, \Gamma=0.01, \Gamma_{S}=0.02$.

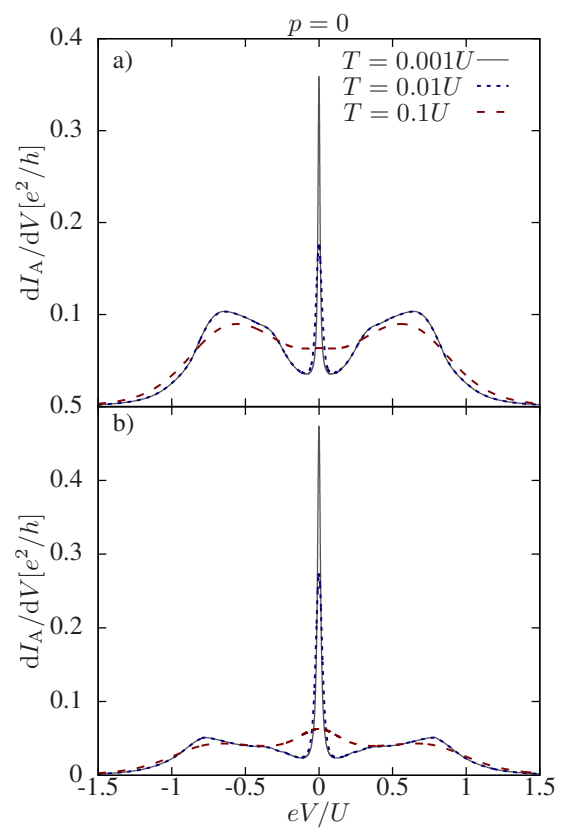

Fig. 2. The differential conductance of the Andreev current as a function of the applied bias voltage for different temperatures and (a) $\varepsilon_{d}=-0.5 U$, (b) $\varepsilon_{d}=$ $-0.33 U$. The other parameters are: $U=0.1 D, \Gamma=$ $0.1 U, \Gamma_{S}=0.2 U$ and $p=0$ (i.e. for nonmagmetic normal lead).

In the case of $\varepsilon_{d}=-U / 2, p=0$ and $T=0.001 U$, $\mathrm{d} I_{\mathrm{A}} / \mathrm{d} V$ exhibits three different peaks, see Fig. 2. The central one is due to the Kondo effect, while the two side peaks resemble Hubbard peaks, but are not as smooth as in the absence of S. In fact, each of them consists of two merged resonances corresponding to the Andreev bound state energies. As can be seen, the Kondo peak is much 


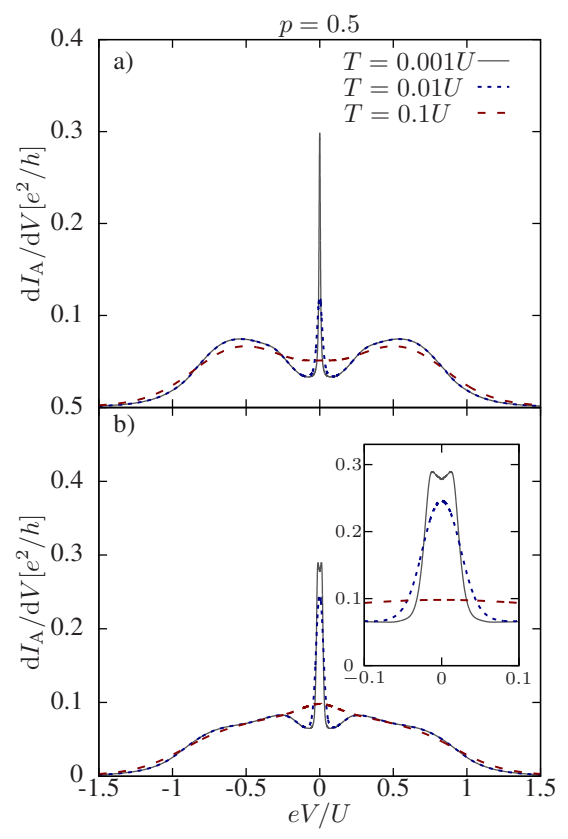

Fig. 3. The same as in Fig. 2 for ferromagnetic lead possessing spin polarization $p=0.5$. The inset in part (b) is an enlargement of low-bias region of the plot.

lower than $4 e^{2} / h$. This is because the parameter $U$ is not chosen in such a way as to compensate for the asymmetry of couplings [9]. Instead of maximizing the Kondo resonance, in this paper we rather focus on the role of spindependent tunneling in the transport properties. With increasing temperature, the Kondo effect becomes suppressed and the side peaks become smoother, see Fig. 2. On the other hand, for $\varepsilon_{d} \neq-U / 2$, the conductance is slightly suppressed, however, the Kondo peak is a bit higher; see Fig. 2b. At high temperatures, however, the Kondo peak at zero bias disappears.

Now let us analyze the effect of ferromagnetic correlations on the Andreev transport through the considered nanostructure. The Andreev differential conductance for $p=0.5$ is plotted in Fig. 3. For $\varepsilon_{d}=-U / 2$, the influence of spin polarization is not at all impressive; $\mathrm{cf}$. Fig. 2a and Fig. 3a. At first sight, one can only observe the decrease of the conductance, including the height of the Kondo peak, which may be attributed to the factor $\left(1-p^{2}\right)$ in Eq. (6). However, a closer look allows one to notice that the side peaks are now even more distorted than for $p=0$. This observation is, naturally, valid only at low temperatures.

The exchange field effects are clearly visible for $\varepsilon_{d}=$ $-0.33 U$, see Fig. 3b. The most striking feature is the splitting of the Kondo peak for $T=0.001 U$, best visible in the inset. The resonance is suppressed at zero bias and has only small side resonances. This is in fact a characteristic feature of the Kondo effect in quantum dots coupled to $\mathrm{F}$ leads $[11,12]$. The split Kondo resonance is visible only at temperatures smaller than the exchange field. With increasing $T$, the Kondo peak becomes first restored (with smaller height), but then eventually dis- appears. We also note that while the split Kondo peak is significantly lower than for $p=0$, the differential conductance for higher voltages is enhanced in comparison with the nonmagnetic case. Moreover, the splitting of the side peaks are now more visible.

In conclusion, we have analyzed the Andreev conductance through a quantum dot strongly coupled to ferromagnetic and superconducting lead. We have shown that the presence of the exchange field due to ferromagnetic lead gives rise to the splitting of the zero-bias Kondo peak at low temperatures, which becomes suppressed with increase of the temperature.

\section{References}

[1] A.F. Andreev, J. Exp. Theor. Phys. 46, 1823 (1964) (Sov. Phys. JETP 19, 1228 (1964)).

[2] J. Bauer, A. Oguri, A.C. Hewson, J. Phys. Condens. Matter 19, 486211 (2007).

[3] T. Domański, A. Donabidowicz, K.I. Wysokiński, Phys. Rev. B 78, 144515 (2008).

[4] S. de Franceschi, L.P. Kouwenhoven, C. Schönenberger, W. Wernsdorfer, Nat. Nanotech. 5, 703 (2011).

[5] J. Kondo, Prog. Theor. Phys. 32, 37 (1964).

[6] D. Goldhaber-Gordon, H. Shtrikman, D. Mahalu, D. Abusch-Magder, U. Meirav, M.A. Kastner, Nature 391, 156 (1998).

[7] M.R. Buitelaar, T. Nussbaumer, C. Schönenberger, Phys. Rev. Lett. 89, 256801 (2002).

[8] R.S. Deacon, Y. Tanaka, A. Oiwa, R. Sakano, K. Yoshida, K. Shibata, K. Hirakawa, S. Tarucha, Phys. Rev. B 81, 121308 (2010).

[9] Y. Tanaka, N. Kawakami, A. Oguri, J. Phys. Soc. Jap. 76, 074701 (2007).

[10] J. Martinek, M. Sindel, L. Borda, J. Barnaś, J. König, G. Schön, J. von Delft, Phys. Rev. Lett. 91, 247202 (2003).

[11] A.N. Pasupathy, R.C. Bialczak, J. Martinek, J.E. Grose, L.A.K. Donev, P.L. McEuen, D.C. Ralph, Science 306, 86 (2004).

[12] M. Gaass, A.K. Hüttel, K. Kang, I. Weymann, J. von Delft, Ch. Strunk, Phys. Rev. Lett. 107, 176808 (2011).

[13] L. Hofstetter, A. Geresdi, M. Aagesen, J. Nygård, C. Schönenberger, S. Csonka, Phys. Rev. Lett. 104, 246804 (2010).

[14] J. Bardeen, L.N. Cooper, J.R. Schrieffer, Phys. Rev. 106, 162 (1957); Phys. Rev. 108, 1175 (1957).

[15] A.V. Rozhkov, D.P. Arovas, Phys. Rev. B 62, 6687 (2000).

[16] Q.-F. Sun, J. Wang, T.-H. Lin, Phys. Rev. B 59, 3831 (1999).

[17] K.G. Wilson, Rev. Mod. Phys. 47, 773 (1975).

[18] We used an open-access Budapest NRG code, see: O. Legeza, C.P. Moca, A.I. Tóth, I. Weymann, G. Zaránd, arXiv:0809.3143 (2008).

[19] A. Freyn, S. Florens, Phys. Rev. B 79, 121102 (2009).

[20] K.P. Wójcik, I. Weymann, Phys. Rev. B 89, 165303 (2014). 